\title{
The role of convection in AGB modeling
}

\author{
Paolo Ventura \\ INAF - Osservatorio Astronomico di Roma Via Frascati 33, 00040 \\ MontePorzio Catone (RM), Italy \\ email: ventura@oa-roma.inaf.it
}

\begin{abstract}
The modeling of the Asymptotic Giant Branch phase is made highly uncertain by some still unsolved issues related to the input macro-physics used to calculate the stellar evolution, namely mass loss, nuclear cross sections, overshooting and convective modeling. We show that in the massive intermediate mass models, which achieve at the bottom of their convective envelope temperatures sufficiently high to favour an advanced nucleosynthesis, the treatment of convection plays a major role in determining the physical and chemical evolution of the stellar models during this evolutionary phase.
\end{abstract}

Keywords. Convection, stars: evolution: AGB

\section{Introduction}

Shortly after the end of the core He-burning phase, stars of intermediate mass $\left(1 M_{\odot} \leqslant\right.$ $M \leqslant 7 M_{\odot}$, hereinafter IMS) evolve through the Asymptotic Giant Branch (AGB) phase. Above a degenerate core made up of carbon and oxygen, a CNO burning shell supplies for most of the time the global energy release; periodically, a He-rich layer below is ignited in conditions of thermal instability, determining what is commonly known as a thermal pulse (TP) (Iben 1975; Iben 1976; Schwartzschild \& Harm 1965; Schwartzschild \& Harm 1967).

During the evolution along the AGB the core mass increases, and the bottom of these stars becomes progressively hotter: in the most massive models $\left(M \geqslant 4 M_{\odot}\right)$ it may attain temperatures sufficiently high to allow an advanced nucleosynthesis, with consequent changes of the surface chemical composition. We refer to this process as Hot Bottom Burning (HBB) (Blöcker \& Schönberner 1991; D'Antona \& Mazzitelli 1996). The HBB mechanism found a solid confirm by the detection of super rich Lithium stars (Abia et al. 1991; Smith \& Lambert 1989; Smith \& Lambert 1990), in which the spectacularly large surface lithium abundances observed are achieved via the Cameron - Fowler mechanism, which requires temperatures at the bottom of the convective zone as large as $\sim 40 \times 10^{6} \mathrm{~K}$ to be activated (Cameron \& Fowler 1971).

Other than HBB, the alternative way of changing the surface chemistry of AGBs is the third dredge-up process (hereinafter TDU). Following the extinction of a TP, the bottom of the outer envelope penetrates inwards, and may reach internal layers precedently involved in the convective shell which is formed shortly after the ignition of $3 \alpha$ reactions: in this case products of He-burning, mainly carbon, are convected to the surface (Iben 1975). Many episodes of TDU would lead to the formation of carbon stars, which have been extensively detected both in the Galaxy and in the Magellanic Clouds (Frogel et al. 1990; Smith et al. 1987).

Recently, the interest towards the AGB evolution raised because they were suggested as a possible explanation of the anomalous chemical composition of some Globular Cluster (GC) stars, whose chemistry, far from being completely homogeneous, shows some interesting and still unexplained patterns (Gratton et al. 2004), the most evident of which 
is the oxygen - sodium anticorrelation, the anomalous stars showing a strong depletion of oxygen accompanied by a sodium enhancement (Carretta 2006). According to the "self-enrichment" scenario hypothesis, the stars showing the anomalous chemistry might have been born when the gas within the cluster was polluted by the evolution of an early generation of IMS, which during their AGB phase reverse into the interstellar medium material changed with respect to the original composition by both HBB and TDU (Ventura et al. 2001). To confirm this idea it is mandatory to build AGB yields which are oxygen poor, and whose $\mathrm{C}+\mathrm{N}+\mathrm{O}$ sum is constant (Ivans et al. 1999).

Understanding the role which AGBs might play requires a detailed and accurate computation of their chemical yields, which, in turn, are extremely sensitive to the details of their physical evolution, mainly to the thermodynamic conditions present at the bottom of their external convective zone.

In this paper we investigate the role played by the modeling of convection on the AGB evolution; we show that when an efficient treatment of convection is adopted, HBB conditions may be easily achieved within massive AGBs. Particularly, we find that an efficient convective modeling favours a strong depletion of oxygen, and, on the physical side, leads to a faster overall AGB evolution, characterized by a small number of TPs: the role of TDU is consequently reduced.

\section{The evolution code}

The evolutionary sequences presented here were calculated by means of the stellar evolution code ATON, a full description of which can be found in Ventura et al. (1998).

\subsection{Convection}

A correct description of the convective regions is one of the biggest challenges for stellar evolution studies. The highly non local character of convection can be described only by means of the Navier - Stokes equation; though some interesting and promising approaches have been suggested (Canuto 1992; Xiong 1985), a full solution of the above equations, to be easily implemented in the stellar evolution codes, is not yet available. Thus, within the context of stellar modelling, convection must be treated non locally, although this renderes the results highly uncertain, particularly in the regions close to the convective boundaries, where non locality effects are expected to be relevant.

The code allows to model convection either via the traditional Mixing Length Theory (MLT, Vitense 1953), or by using the Full Spectrum of Turbulence model (hereinafter FST) developped by Canuto \& Mazzitelli (1991). The interested reader may find a complete analysis of the physical differences among the two models in Canuto \& Mazzitelli (1991). In this paper we explore the role played by convection comparing results obtained with the FST model with those found with an MLT treatment.

\subsection{Mixing}

Within massive AGBs, a non-negligible fraction of the global energy release is generated within the outer convective envelope (Blöcker \& Schönberner 1991), thus requiring the use of a diffusive algorythm, in which nuclear burning and mixing of chemicals are treated simultaneously (Cloutman \& Eoll 1976). The borders of the convective regions are determined via the classic Schwartzschild criterium, which allows to find the point where buoyancy vanishes; the distance beyond the border that convective eddies travel within the stability region, the overshooting distance, is usually parametrized, extending artificially the region which is chemically mixed. Within the context of the diffusive approach, overshooting is modeled allowing an exponential decay of convective velocities within 
the radiative regions (Herwig et al. 1997), with an e-folding distance parametrized by $\zeta H_{p}$. This behaviour of convective velocities is also confirmed by numerical simulations (Freytag et al. 1996).

In this work, extra-mixing from the convective core during the core $\mathrm{H}$ - and He-burning phases was modeled by assuming $\zeta=0.02$, according to a calibration given in Ventura et al. (1998). No extra-mixing was assumed from the bottom of the convective envelope during the whole evolutions. The consequences on the physical evolution and on the chemical yields of AGBs determined by the assumption of some extra-mixing from the bottom of the convective envelope was investigated by Ventura \& D'Antona (2005a).

\subsection{Mass loss}

Mass loss is a key-ingredient for the AGB evolution, because the stars suffer a strong mass loss during this evolutionary phase, and the AGB evolution itself is eventually halted when the mass of the envelope is significantly reduced. The rate of mass loss adopted determines also the degree of nucleosynthesis which may be achieved at the bottom of the convective envelope, and has thus a relevant effect on the chemical composition of the ejecta. In this work we modeled mass loss following the prescription given by Blöcker (1995); the free parameter entering the quoted formula was set to $\eta_{R}=0.02$, according to a previous calibration based on observations of lithium rich AGBs within the Magellanic Clouds given in Ventura et al. (2000).

\subsection{Nuclear network and cross sections}

The nuclear network includes 30 elements (up to ${ }^{31} \mathrm{P}$ ) and 64 reactions. A detailed description can be found in Ventura \& D'Antona (2005b). The cross sections adopted were taken from the NACRE compilation (Angulo et al. 1999). The models have a chemical composition typical of $\mathrm{GCs}$, i.e. $\mathrm{Z}=0.001$ and $\mathrm{Y}=0.24$. All the abundances are solar scaled.

\section{AGB evolution}

To investigate the role played by convection on AGB modeling, we compare the evolutions of three $5 M_{\odot}$ models, differing only in the treatment of convection, i.e. FST and MLT; in this latter case the free parameter providing the mixing length was chosen as $\alpha=1.7$ ( $\alpha 1.7$ model, the value needed to simulate the evolution of the Sun), and $\alpha=2.1$ $(\alpha 2.1$ model $)$.

\subsection{The physical and chemical evolution}

In the left panel of Fig. 1 we compare the temporal variation of the luminosity of the three afore mentioned models; the right panel shows the temperature $\left(\mathrm{T}_{\mathrm{bce}}\right)$ at the bottom of the convective envelope, in $10^{6} \mathrm{~K}$ units. In all cases, both luminosity and $\mathrm{T}_{\mathrm{bce}}$ first increase with time, reach a maximun, then decline when the mass of the envelope is significantly reduced. The tight correlation between luminosity and $\mathrm{T}_{\mathrm{bce}}$ is due to the fact that in massive AGBs a considerable fraction of the luminosity is generated within the convective envelope (Blöcker \& Schönberner 1991).

We note from Fig.1 that the FST model (solid track), compared to MLT, evolves at larger luminosities and $\mathrm{T}_{\mathrm{bce}}$ 's; the MLT results depend critically on the value chosen for $\alpha$. This behaviour has two important evolutionary consequences on the models:

(a) The AGB life-time of the FST model is shorter, due to the strong dependence of mass loss on luminosity. 

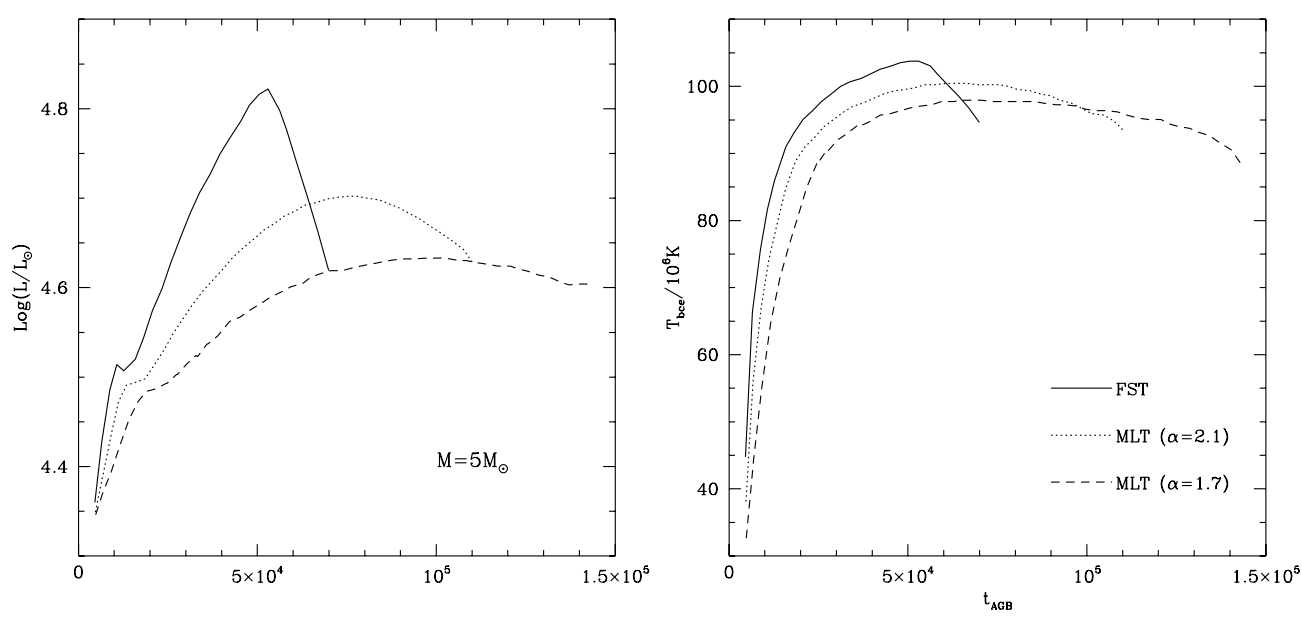

Figure 1. The variation with time of luminosity (Left) and temperature at the bottom of the convective envelope (Right) of the three evolutionary sequences discussed in the text, differing for the modeling of convection. Time is counted starting from the beginning of the AGB phase.
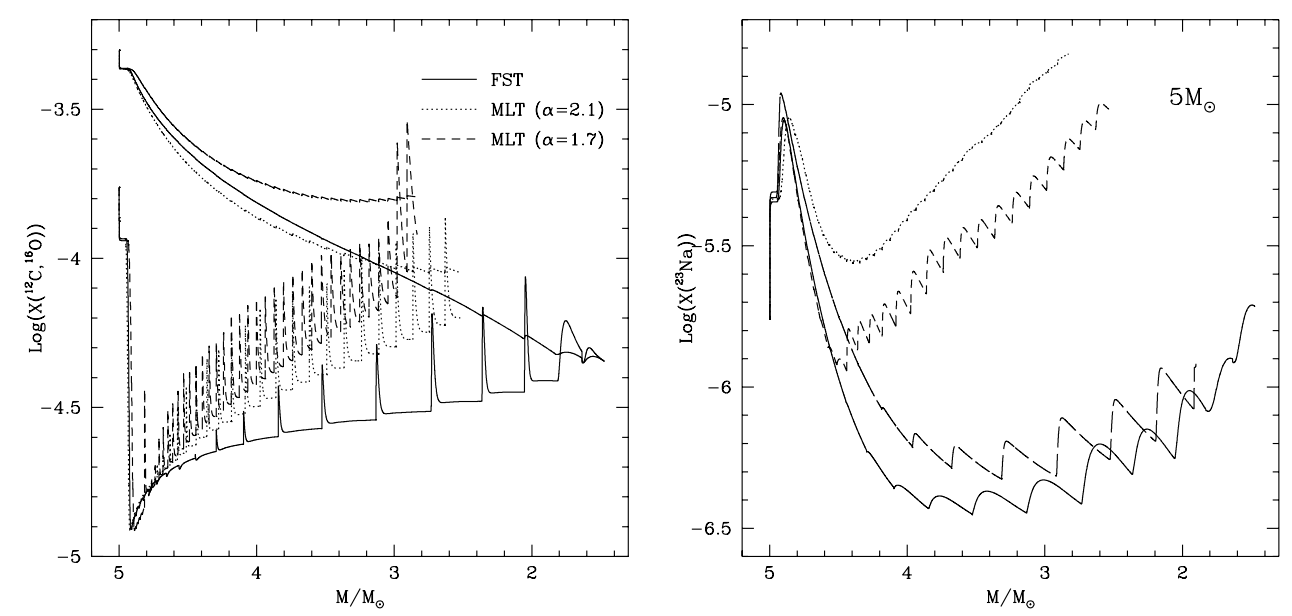

Figure 2. Variation during the AGB phase of the surface abundances of carbon and oxygen (Left) and of sodium (Right) for the same three models presented in Fig.1. The dotted-dashed line in the right panel indicates the results obtained with FST and taking the limits of the NACRE reaction most favouring sodium production.

(b) The number of TPs, and consequently of possible TDU's, is much higher when the MLT treatment is adopted.

For that concerning the chemical content of the ejecta, we show in Fig.2 the variation of the surface abundances of carbon and oxygen (left) and sodium (right) in the three cases; we report the total mass on the abscissa, to have an idea of the yields of these elements.

The strong depletion of oxygen found in the FST case, compared to the modest reduction observed in the $\alpha 1.7$ model, is a clear indicator of the different degree of nucleosynthesis reached in the various cases. The differences in the sodium content shown in the right panel of Fig.2 are even more critically dependent on the convective modeling. In the 
FST case, sodium is destroyed after the first TPs, due to the high temperatures at the bottom of the envelope, favouring proton capture; this effect is clearly overwhelming the following production due to proton capture by ${ }^{22} \mathrm{Ne}$ nuclei dredged-up after each TP, so that we find a global sodium yield smaller than the initial abundance. On the contrary, in the MLT cases, particularly when $\alpha=1.7$ is used, the sodium depletion is very modest, and eventually the effects of many dredge-up episodes raise the sodium abundance, so that the final yield exceeds the initial value by almost two orders of magnitude. To have an idea of how much the results are sensitive to the convective model adopted, we show in the right panel of Fig.2 the surface abundance of sodium of an FST model where the sodium production is favoured, by choosing the upper limits for the reactions producing sodium, and the lower limits for the $(p, \alpha)$ and $(p, \gamma)$ proton capture reactions by sodium nuclei: we see that the surface chemistry of this class of objects is by far more dependent on the convective modeling than on the uncertainties associated to the nuclear cross sections.

Though we show the surface abundances of only three elements, we may infer that the surface chemistry of massive AGBs depend on the modeling of convection as follows:

(a) Use of the FST treatment leads to a much more advanced nucleosynthesis, due to strong HBB.

(b) In the FST case the full CNO cycle is easily activated, leading to a strong depletion of oxygen; this latter is made difficult by the use of the MLT modeling, expecially in the $\alpha 1.7$ model.

(c) TDU is found much more easily with the MLT treatment, because it evolves at lower luminosities, thus experiencing a larger number of TPs. In the FST case, either TDU is not achieved, or it is found only at the latest evolutionary stages. Thus, we expect a much larger surface enrichment of carbon in the MLT case, together with an overall increase of the $\mathrm{C}+\mathrm{N}+\mathrm{O}$ sum.

\subsection{The thermodynamic structure of the bottom of the envelope}

We show in the top panel of Fig.3 the temporal variation of the luminosity of the three models discussed in the present investigation in the early AGB phase, during the first three TPs. The bottom panel shows the corresponding evolution of $\epsilon_{n}^{\max }$, the maximum internal value of the coefficient $\epsilon_{n}$ for the generation of nuclear energy. We note that the differences among the luminosities of the three models arise already in the early AGB phase, though the structure of the layers where the peak of CNO burning energy release is located is very similar in the three cases, as we may understand by comparing the values of $\epsilon_{n}^{\max }$. We conclude that when convection is modeled by FST, more nuclear energy is generated in the regions external to the CNO burning peak, which are close (or inside) to the outer convective zone.

Understanding these differences requires a detailed inspection of the thermodynamic stratification of these regions of the star.

We focus our attention on the peak of luminosity reached after the second TP, $\sim 9000 \mathrm{yr}$ after the beginning of the AGB evolution. We compare in the two panels of Fig. 4 the internal profiles of the radiative gradient $\nabla_{\mathrm{rad}}$ (left), and of $\epsilon_{\text {nucl }}$ in the three cases. We see from the left panel that, consistently with the high efficiency of the FST model, which tends to enhance the fraction of flux carried by convection, the $\nabla_{\text {rad }}$ profile is steeper $\left(F_{\mathrm{rad}} \propto 1 / \nabla_{\mathrm{rad}}\right)$ within the convective region, which leads to a more internal location of the convective boundary. This, in turn, has the effect of carrying ${ }^{3} \mathrm{He}$ rich material in hotter regions, thus triggering an extra-luminosity, which can be detected in the right panel as a small discontinuity in the profile of $\epsilon_{\text {nucl }}$. 


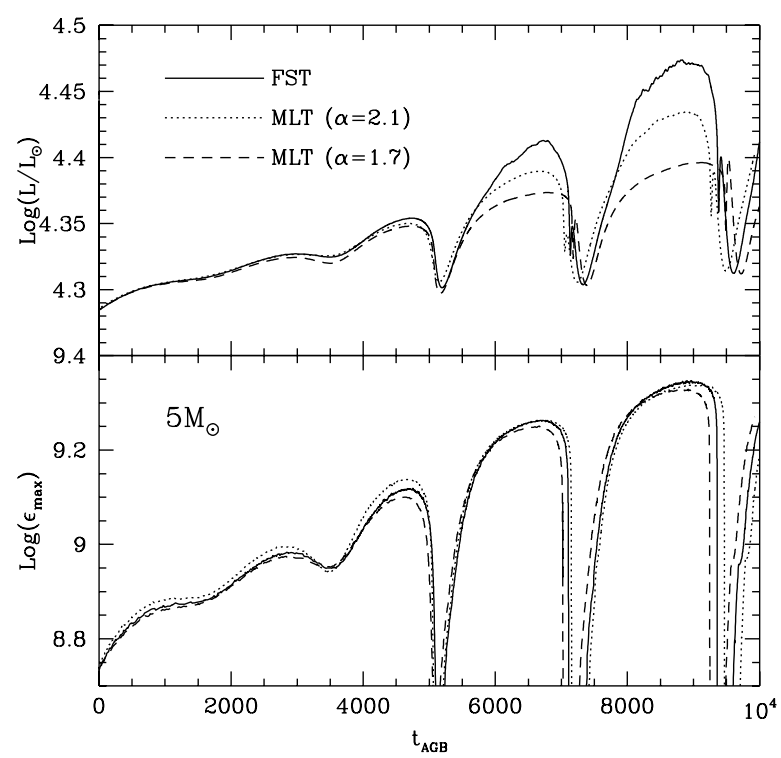

Figure 3. Top: Variation with time of the luminosity of the three models presented in Fig.1 during the first Thermal Pulses. Bottom: The temporal variation of the maximum internal value of the coeffiecient for the generation of nuclear energy.
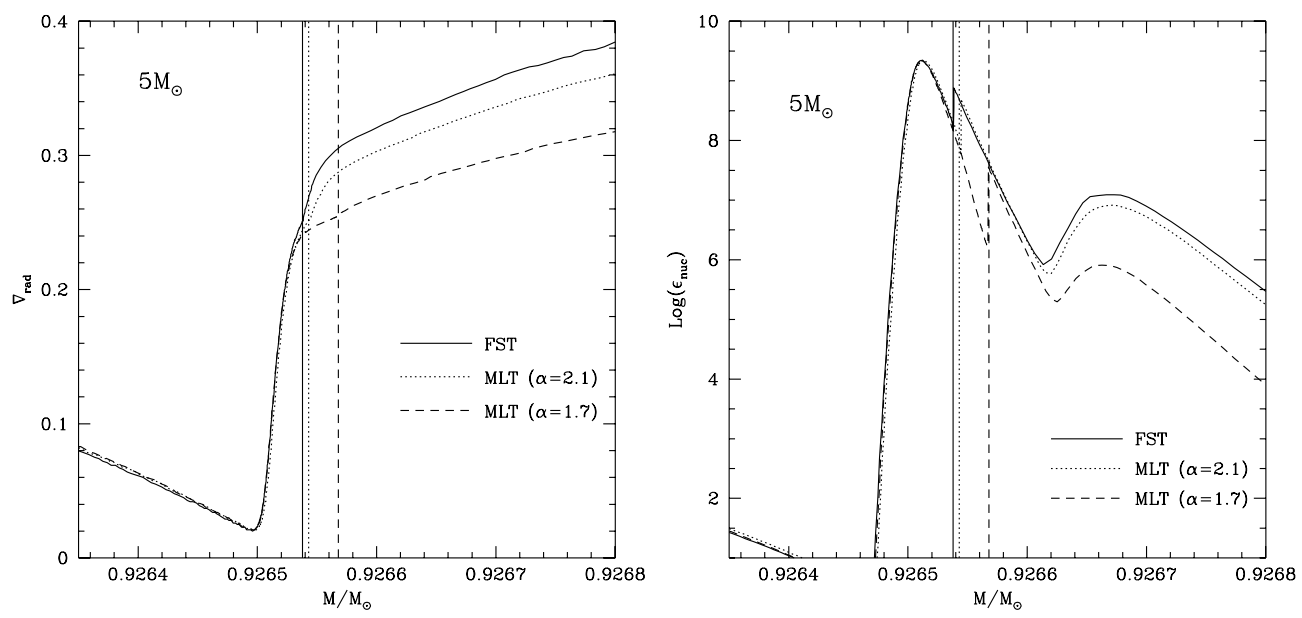

Figure 4. The internal distributions of the radiative gradient (Left) and of the coefficient for the generation of nuclear energy (Right) in the three models calculated with various convective models. The profiles refer to the stage of maximum luminosity following the second TP. The vertical lines mark the bottom of the convective envelope.

\section{Conclusions}

We investigate the role played by the treatment of convection in the modeling of the AGB phase of IMS, focusing our attention on the more massive models, those achieving HBB conditions at the bottom of their convective envelope.

We show that when an efficient treatment of convection (like the FST model) is used, $\mathrm{HBB}$ conditions are reached much more easily, which makes the models to evolve at larger 
luminosities, with the consequence that the overall AGB life time becomes shorter, and the number of TPs is reduced.

On the chemical point of view, in the FST case the yields show the signatures of an advanced nucleosynthesis, with a strong oxygen depletion. On the other hand, in the MLT case many more TDU episodes are expected, thus the ejecta are more rich of ${ }^{12} \mathrm{C}$ : the total $\mathrm{C}+\mathrm{N}+\mathrm{O}$ sum is expected to largely exceed unity. Very large differences are expected for the abundances of those elements, e.g. sodium, whose surface abundance depends both on HBB and on TDU. The FST model, favouring oxygen destruction and constant $\mathrm{C}+\mathrm{N}+\mathrm{O}$, produces chemical yields of massive AGBs consistent with the selfenrichment scenario of GCs.

The convective modeling has a strong impact on both the physical and chemical evolution of AGB models, and the uncertainties related to the treatment of convective zones must be considered when evaluating the predictive power of the results obtained.

\section{References}

Abia, C., Boffin, H.M.J., Isern, J., \& Rebolo, R. 1991, A\& A 245, L1

Angulo, C. 1999, Nucl. Phys. A, 656, 3

Blöcker, T. 1995, A\&̈A 297, 727

Blöcker, T. \& Schönberner, R. 1991, A\&A 244, L43

Cameron, A.G. \& Fowler, W.A. 1971, ApJ 164, 111

Canuto, V.M. 1992, ApJ 389, 742

Canuto, V.M. \& Mazzitelli, I. 1991, ApJ 370, 295

Carretta, E. 2006, AJ 131, 1766

Cloutman, L. \& Eoll, J.G. 1976, ApJ 206, 548

D'Antona, F. \& Mazzitelli, I. 1996, ApJ 470, 1093

Freytag, B., Ludwig, H.G., \& Steffen, M. 1996, A\&SA 313, 497

Frogel, J.A., Mould, J., \& Blanco, V.M. 1990, ApJ 352, 96

Gratton, R., Sneden, C., \& Carretta, E. 2004, ARAA 42, 385

Herwig, F., Blöcker, T., \& Schönberner, D. 1997, A\&A 324, L81

Iben, I.J. 1975, $A p J$ 196, 525

Iben, I.J. 1976, ApJ 208, 165

Ivans, I.I., Sneden, C., Kraft, R.P., et al. 1999, AJ 118, 1273

Schwartzschild, M. \& Harm, R. 1965, ApJ 142, 855

Schwartzschild, M. \& Harm, R. 1967, ApJ 150, 961

Smith, V.V., Lambert, D.L., \& McWilliam, A. 1987, ApJ 320, 862

Smith, V.V. \& Lambert, D.L. 1989, ApJ 345, L75

Smith, V.V. \& Lambert, D.L. 1990, ApJ 361, L69

Ventura, P., \& D'Antona, F. 2005, ApJ 635, L149

Ventura, P., \& D'Antona, F. 2005, ApJ 431, 279

Ventura, P., D’Antona, F., \& Mazzitelli, I. 2000, A\&A 363, 605

Ventura, P., D'Antona, F., Mazzitelli, I., \& Gratton, R. 2001, ApJ 550, L65

Ventura, P., Zeppieri, A., D’Antona, F., \& Mazzitelli, I. 1998, A\& A 334, 953

Vitense, E. 1953, ZfA 32, 135

Xiong, D.R. 1985, A\&A 150, 133

\section{Discussion}

J. LATtAnzio: We have some preliminary abundances for two red giants in the SMC $\left(M \sim 5 M_{\odot}\right)$ and they show $\mathrm{C}+\mathrm{N}+\mathrm{O}$ up at least $\sim 1$ dex. Maybe such observations can help discriminate between the different models of convection? 
P. Ventura: The SMC metallicity is larger than that of the models presented here, thus allowing some $\mathrm{C}+\mathrm{N}+\mathrm{O}$ increase even within the FST context. Also, FST models with a very tiny amount of extra-mixing are compatible with a $\mathrm{C}+\mathrm{N}+\mathrm{O}$ increase, even at these low metallicities. Thus, any discrimination among convective models based on this information would be highly uncertain.

I. RoxbuRGH: If I understood correctly you did not include overshooting (or undershooting). If you did, could you reproduce the results of the Canuto-Mazzitelli theory using MLT with undershoot?

P. VenturA: I couldn't. When undershoot is included, the only physical difference is that thermal pulses are ignited later, because more helium needs to be accumulated in the H-exhausted region; yet, the evolution of luminosity and temperature at the bottom of the envelope remain unchanged.

L. Deng: As you have implemented Canuto \& Mazzitelli (1991) Theory into your evolution code, have you ever found supersonic convection in your AGB model ?

P. Ventura: No. I didn't. Anyway, this study is focused on the thermodynamic properties of the bottom of the envelope, thus the structure of the outermost layers was not examined carefully. 